\title{
Una mirada de la cultura organizacional y su incidencia en la competitividad de las organizaciones en el contexto global
}

\author{
Isabel Regina Armas Heredia \\ Universidad Técnica de Cotopaxi, Latacunga, Ecuador \\ Universidad de Los Andes, Programa de Doctorado en Ciencias Contables, \\ Mérida, Venezuela \\ Correo electrónico del autor: isabel.armas@utc.edu.ec \\ Recepción: 12 Octubre 2017 \\ Aprobación: 7 Diciembre 2017
}

\section{Resumen}

En el campo administrativo, la globalización se entiende como un proceso que ha introducido transformaciones relevantes en el funcionamiento de las organizaciones. Tales cambios, revelan la importancia estratégica adquirida por la dimensión cultural como base del desarrollo organizacional, pues a partir de ésta se promueve un mejoramiento en el desempeño, la productividad y la competitividad empresarial en los mercados globales. En esta dirección, el artículo tiene como objetivo analizar la incidencia de la cultura corporativa en la competitividad de las organizaciones en el contexto global. Para el alcance de este objetivo, metodológicamente se procedió a formular un diseño investigativo basado en la revisión y análisis de literatura en los campos de la globalización, y la cultura organizacional, así como de su incidencia en el desarrollo de las organizaciones. El trabajo se estructuró en tres secciones: en la primera, se aborda el tema de la globalización enfatizando en sus implicaciones en el desarrollo de las organizaciones; en la segunda, se diserta sobre la cultura organizacional como elemento clave que contribuye con el desarrollo de las organizaciones en el contexto global y en la tercera, se presentan las conclusiones destacando la importancia estratégica adquirida por la cultura corporativa como elemento que contribuye con el mejoramiento del desempeño y la competitividad de las organizaciones en los mercados globalizados.

Palabras clave: globalización, organizaciones, cultura organizacional, desarrollo organizacional, competitividad.

REVISTA DE INVESTIGACIÓN SIGMA / Vol. 04, No 1, 2017 (pág. 101-114) 


\title{
A look at the organizational culture and its impact on the competitiveness of organizations in the global context
}

\begin{abstract}
In the administrative field, globalization is understood as a process that has introduced relevant transformations in the functioning of organizations. Such changes reveal the strategic importance acquired by the cultural dimension as a basis for organizational development, since it promotes an improvement in performance, productivity and business competitiveness in global markets. In this direction, the article aims to analyze the impact of corporate culture on the competitiveness of organizations in the global context. To reach this objective, methodologically, a research design was developed based on the review and analysis of literature in the fields of globalization, and the organizational culture, as well as its impact on the development of organizations. The work was structured in three sections: in the first, the topic of globalization is addressed, emphasizing its implications for the development of organizations; In the second, the organizational culture is discussed as a key element that contributes to the development of organizations in the global context and in the third, conclusions are presented highlighting the strategic importance acquired by the corporate culture as an element that contributes to the improvement of performance and competitiveness of organizations in globalized markets.
\end{abstract}

Keywords: globalization, organizations, organizational culture, organizational development, competitiveness.

\section{Introducción}

La globalización se concibe como un proceso que ha inducido transformaciones profundas en las dimensiones sociales, económicas, institucionales, culturales y ambientales que se articulan para promover el desarrollo económico y social en los diversos países. En tal sentido, el funcionamiento de las organizaciones no está ajeno al proceso global, pues se han introducido cambios trascendentes en los modelos de gestión para el alcance de la competitividad de las empresas en los mercados globales.

Comopartedeloscambiosexperimentados por los modelos de gestión organizacional, destaca el paso de las formas organizativas jerárquicas, con estructura centralizada, basadas en la división del trabajo, en las cuales la cultura organizacional no se configuraba como un aspecto clave del desarrollo organizacional; hacia nuevas formas de organización caracterizadas básicamente por una estructura descentralizada, la promoción del trabajo en equipo para la gestión del conocimiento, la participación de sus miembros en la toma decisiones, así como la conformación de una cultura empresarial como base del desarrollo organizativo.

Considerando los argumentos expuestos, este artículo tiene como objetivo analizar la 
incidencia de la cultura organizacional en la competitividad de las organizaciones en el contexto de la globalización. Para el desarrollo del mismo, se ha seguido una metodología basada la revisión de literatura especializada en las líneas de investigación relacionadas con la globalización, y la cultura organizacional.

El artículo se ha estructurado en tres secciones. En la primera, se diserta en torno a la globalización y sus efectos en el desarrollo organizacional, con especial énfasis en los fundamentos quehan generadolahomogenización de las prácticas y estructuras organizativas como es el caso de las denominadas Megaempresas; en la segunda, se aborda la influencia de la cultura en el desarrollo organizacional, precisando los aportes de la dimensión cultural a la competitividad de las organizaciones en los mercados globalizados; finalmente en la tercera, se presentan las conclusiones del estudio enfatizándose en la importancia de la cultura organizacional para el mejoramiento de la competitividad empresarial, lo cual se alcanza en la medida que los miembros de la organización se identifican con los valores, intereses, objetivos y prácticas que conforman la dimensión cultural de la organización.

El contexto global como base del actividades productivas, los modelos de gestión desarrollo organizacional
El término globalización se entiende como un proceso de transformación de las diversas dimensiones que conforman el desarrollo. Concretamente en el campo de las organizaciones, a partir de este proceso se ha generado un cambio en las estructuras económicas, sociales, culturales, institucionales y tecnológicas que rigen su funcionamiento en el contexto global.

Al respecto Ritzer (2007), define la globalización como un proceso de cambio estructural que enfatiza en la difusión de las prácticas, el incremento de las relaciones comerciales entre las naciones, la existencia de nuevas formas de organización de la vida social, así como la existencia de una conciencia socialmente compartida.

Sobre la base de esta definición, se deduce que las implicaciones de la globalización en el desarrollo de las organizaciones, no solamente se cambios introducidos a nivel de los sistemas de producción, se ha presentado transformaciones de carácter social, cultural e institucional que han inducido transformaciones en cuanto a las relaciones sociales establecidas en el marco de las presentan a nivel económico, pues además de los 
organizacional, así como los fines y metas de la organización.

En esta dirección Ritzer (2007), plantea que la globalización se gesta a partir de la teoría de la modernidad, bajo la cual se destaca el papel desempeñado por las organizaciones capitalistas, así como de los Estados modernos, en la promoción del desarrollo económico y social. Para el autor, en esta concepción de lo global destacan los aportes de la teoría marxista así como de la teoría weberiana.

Desde la postura marxista, la principal fuerza que impulsa la globalización se encuentra la necesidad de las corporaciones de obtener niveles de ganancias crecientes a partir de la existencia de un imperialismo económico de amplio alcance, así como la necesidad de las empresas, instituciones y del Estado de promover una hegemonía cultural a nivel mundial; mientras que desde la posición de Weber, se enfatiza en la diseminación y aplicación de las estructuras racionalizadas a nivel global.

Concretamente a nivel cultural, Ritzer (2007) plantea que con la globalización, se ha generado una homogenización de los códigos y prácticas comunes que rigen el funcionamiento de las organizaciones a nivel global. Esta tendencia se asocia directamente "con la influencia internacional creciente de una cultura particular" (p.133).

Bajo esta postura, las grandes corporaciones han fundamentado su expansión a nivel global, a partir de la aplicación de modelos de gestión basados en la promoción de un una estructura homogénea en la organización en cuanto a servicios, productos, sistemas de producción y dirección, entre otros aspectos. Por ende, se puede afirmar que estas empresas han impulsado la conformación de una cultura organizacional homogénea como base de su desarrollo a nivel global.

Como ejemplo de los efectos de la globalización en el campo de las organizaciones, Ritzer (2007) expone el caso de la McDonalización, definido en los términos de un modelo de gestión caracterizado por la eficiencia en el servicio prestado al cliente; la calculabilidad de los tiempos de producción y de entrega; la predecibilidad y el control de las actividades y funciones; el desarrollo de procesos continuos de innovación y desarrollo tecnológico, así como la creciente racionalidad formal y la burocratización.

El proceso descrito, ha fundamentado la 
expansión de las franquicias de comida rápida tanto en la sociedad americana, como a nivel mundial. A juicio de Ritzer (2007) esta franquicia se perfila como el prototipo de la racionalización y homogenización promovida por la globalización a nivel mundial.

En este contexto, Esteves (2000) señala que a partir de la década de los años noventa, se ha generado un proceso de internacionalización que ha conducido a lo que se ha denominado mundialización de la economía, caracterizado básicamente por las fusiones de empresas monopólicas u oligopólicas (Megaempresas), de distintos países que anteriormente competían en el mercado.

Paralaautora,elprocesodemundialización de la economía ha traspasado las fronteras de los países de origen de las Megaempresas, pues éstas se han insertado en el contexto global con el fin de dominar los mercados internacionales. Como ejemplo de Megaempresas a nivel mundial, Esteves (2000) cita los casos de las siguientes fusiones:

1. En el campo automotriz: Chrysler (norteamericana) con Daimler-Benz (alemana) y; Volvo (sueca) con Renault (francesa).
2. En el área petrolera: Total Fina (belga) con la Elf Aquitaine (francesa).

3. En el sector farmacéutico: Glaxo Wellcome (Gran Bretaña) y Smith Kline Beecham (Estados Unidos).

4. En el sector financiero: se creó la International Exchanges (IX) a partir de la fusión de la Bolsa de Valores de Londres con la Bolsa de Valores de Francfort (Alemania).

Sobre la base de lo expuesto, se puede afirmar que la globalización se manifiesta en las dimensiones económicas, administrativas, técnicas, institucionales y culturales que fundamentan el desarrollo de las organizaciones en el escenario global, lo cual ha conducido a una homogenización de las prácticas, tecnológicas, sistemas de producción, incluyendo las tecnologías de información y comunicación.

La homogenización generada en el campo de las organizaciones a partir de la globalización, se evidencia en el campo contable a través de la adopción de las Normas Internacionales de Información Financiera (NIIF), las cuales se han formulado para impulsar la utilización de un mismo lenguaje en la presentación y revelación de la información financiera de las organizaciones, 
facilitando en consecuencia la toma de decisiones por parte de los usuarios externos e internos, y promoviendo la competitividad de las empresas a nivel internacional.

A partir de este proceso, las empresas al momento de realizar negociaciones internacionales como por ejemplo fusiones para conformar Megaempresas, revelarán la información contable requerida bajo el lenguaje NIIF, evitando tener que preparar la información financiera bajo los Principios Contables que rigen en sus países de origen, así como de los exigidos por los países en los que la corporación aspira operar.

Otros aspectos que revelan la homogenización inducida por la globalización a nivel de las organizaciones, se encuentran en la comercialización de los mismos bienes a nivel mundial aun cuando sus precios pueden varían, así como el establecimiento de relaciones comerciales virtuales para la compra de bienes y la prestación de servicios a través de las tecnologías de información y comunicación (internet, correo electrónico, entre otros.)

Así mismo, otro efecto de la globalización en el desarrollo organizacional se percibe en el papel estratégico que ha comenzado a tener la cultura organizacional en la competitividad de las organizaciones, lo cual es una muestra de los cambios introducidos en los modelos de gestión de las organizaciones a nivel mundial. Tal aspecto, constituye el objeto de la siguiente sección.

\section{La cultura organizacional y su}

\section{influencia en la competitividad de las organizaciones}

En las últimas décadas, el término "cultura organizacional" también conocido como "cultura corporativa" ha comenzado a tener una importancia estratégica en el pensamiento administrativo, pues ésta se considera una de las dimensiones más importantes que influye de manera determinante en el desarrollo de las organizaciones, en la medida que promueve una mejora en la productividad y rendimientos de las mismas.

Desde esta postura, la cultura organizacional se configura como un aspecto complejo que condiciona el funcionamiento de las organizaciones, lo cual trasciende los límites del pensamiento administrativo para ubicarse a un nivel interdisciplinario, cuyo abordaje requiere de los aportes provenientes de diversas disciplinas entre las que destacan la psicología, la 
sociología, la administración, la economía, entre otras. Es decir, la interdisciplinariedad se perfila como un mecanismo clave que promueve el desarrollo económico y social de la organización en sentido amplio.

A partir de la revisión de literatura especializada, Rodríguez (2009) plantea que el interés por el estudio del tema de la cultura organizacional se inicia a partir de la década de los años ochenta, como consecuencia de las diferencias de productividad encontradas al comparar el modelo de gestión empresarial norteamericano y el japonés. La evaluación de ambos modelos, mostró que el desempeño de las organizaciones japonesas en cuando a calidad, y funcionalidad de sus productos era el resultado de la cultura organizacional, lo cual constituyó la base para considerar la dimensión cultural como un activo estratégico de las organizaciones.

Con relación al término cultura, el Diccionario de la Real academia Española (2017), la define como el "conjunto de modos de vida y costumbres, conocimientos y grado de desarrollo artístico, científico, industrial, en una época, grupo social, entre otros." Esta conceptualización, se fundamenta en los aspectos antropológicos de la cultura, pues se enfatiza en los valores y creencias compartidas que forman parte del modo de vida de los grupos sociales. Así mismo, el Diccionario citado define las organizaciones como una "Asociación de personas regulada por un conjunto de normas en función de determinados fines". A partir de ambos conceptos, se puede definir la cultura organizacional como el conjunto de valores, principios e intereses socialmente compartidos por los miembros de una organización.

Tal postura encuentra sus bases en el planteamiento de Rodríguez (2009), bajo el cual se abordan las organizaciones como "pequeñas sociedades con características culturales y con capacidad para crear valores y significados" (p.68). Esta concepción de las organizaciones, vincula directamente su desarrollo con una dimensión cultural que promueve el mejoramiento de su desempeño y el logro de los objetivos planteados.

Al respecto Cújar, Ramos, Hernández y López (2013), plantean que el concepto de cultura en el campo de las organizaciones, tiene sus bases en los aportes de la Escuela de las Relaciones Humanas de la Administración, la cual promovió un conjunto de investigaciones para determinar el efecto de los factores ambientales 
y las condiciones físicas sobre el desarrollo del trabajo.

Posteriormente Schwartz y Davis (citados por Cújar, Ramos, Hernández, 2013) definieron la cultura organizacional como el conjunto de creencias y expectativas compartidas por los miembros de la organización, las cuales conducen al establecimiento de normas informales y no escritas que orientan su conducta y comportamiento para el alcance de los objetivos organizacionales. Como lo expresa Chiavenato (2006), la cultura se manifiesta en las organizaciones a partir de un “conjunto de hábitos, creencias, valores, tradiciones, interacciones y relaciones sociales típicos de cada organización" (p. 319).

Puede afirmarse entonces, que la cultura organizacional se desarrolla a partir de las relaciones que establecen sus miembros, las cuales fundamentan la formulación de un conjunto de objetivos, valores, creencias e intereses socialmente compartidos. Estos se incorporan en la cultura corporativa de la organización, pues contribuyen de manera determinante con su desempeño y desarrollo.

En esta orientación Vargas (2007), define la cultura organizacional a partir de los valores, principios, normas, conocimientos de los procesos productivos, entre otros aspectos que rigen el funcionamiento de la organización. Fundamentándose en esta conceptualización, Cantillo y Daza (2011) afirman que la cultura en las distintas organizaciones constituye la base para la conformación de esquemas valorativos entendidos como un sistema de relaciones que se establece entre las características de la organización y los actores que la conforman (empresarios, gerentes, empleados, entre otros.)

Sobre la base de lo expuesto, cabe mencionar que a partir de la cultura organizacional se ha inducido una transformación relevante en los esquemas organizativos, lo cual a juicio de Lanz (2001) se asocia directamente con el "clima cultural de la Modernidad, a las creencias imperantes, al sentido común dominante, al predominio de los prototipos racionales de la civilización del capital" (Lanz, p. 162). Desde esta postura, la posmodernidad se concibe como la base para la conformación de nuevas prácticas sociales fundamentadas en la cultura, emergiendo en consecuencia nuevas formas organizativas, que en líneas generales presentan una cultura organizacional caracterizada por Rodríguez (2009) en los siguientes términos: 
1. Sistema complejo que tiene una influencia determinante sobre el comportamiento del grupo social que forma parte de la organización.

2. Construcción social (no legalizada) conformada a partir de las relaciones sociales establecidas por los miembros de la organización.

3. Activo intangible e inmaterial basado en los sistemas de comunicación existentes en la organización que contribuye con el mejoramiento del desempeño de la organización.

4. Carácter subjetivo, pues depende de la percepción y postura del observador.

5. En todas las organizaciones, las creencias, valores, intereses y objetivos no siempre resultan ampliamente compartidos y aceptados por todos los miembros de la organización.

Las características descritas se asocian con la cultura de las organizaciones posmodernas, en las cuales no existe un mecanismo de causalidad lineal que se traduzca en una forma pura de organizaciones posmodernas, pues éstas son el producto de una "hibridez de racionalidades, como mezclas de formas, como superposición de esquemas funcionales" (Lanz, 2001, p. 164). Por ende, no existen en el campo empírico formas funcionales de organizaciones posmodernas, lo que existen son formas organizativas en las que convergen las prácticas administrativas del pensamiento tradicional con nuevas formas emergentes de organización (Lanz, 2001).

En tal sentido, resulta de interés destacar que bajo el pensamiento administrativo clásico, las organizaciones se gestionaron siguiendo las bases del modelo burocrático caracterizado básicamente por la división del trabajo, así como de tareas y funciones, la estructura vertical jerarquizada, la toma de decisiones centralizada, la inexistencia de liderazgo y motivación de los empleados, la ausencia de procesos de capacitación, entre otros aspectos. En este caso, la cultura organizacional no se consideró como una dimensión estratégica para promover el desarrollo de las organizaciones.

Posteriormente con la evolución del pensamiento administrativo, las organizaciones han adoptado nuevos modelos de gestión caracterizados por la descentralización, la gestión del conocimiento en el desarrollo organizacional, la participación del capital humano en la toma de 
decisiones, la importancia de la calificación del capital humano, así como la incorporación de los valores e intereses de los miembros como parte de los objetivos organizacionales, mismas que adicionalmente ayudan a lograr una gestión de marketing eficaz que como dice Iturralde (2015), posicionen a la organización en el mercado relacionado a su target.

En esta dirección Lash (2005) plantea que en el contexto de la transformación de los modelos de gestión ha surgido una nueva forma organizativa conocida como desorganización. Para el autor, las desorganizaciones se definen como formas particulares de organización estructuradas a partir de un conjunto de valores. Estas formas organizativas, aun cuando se perfilan como formas particulares de asociación, no presentan una estructura formal e informal, su funcionamiento no está regulado a partir de normas, sino por medio de valores y se presentan dispersas en el plano global. Como ejemplo de desorganizaciones Lash (2005) cita el caso de las asociaciones laborales conformadas en el campo de la biotecnología, software y multimedia.

En esta orientación Lash (2005) distingue las organizaciones de las desorganizaciones en los siguientes términos:
1. Las organizaciones fundamentan su desarrollo a partir del cumplimiento de normas, mientras que las desorganizaciones se desempeñan en función de los valores.

2. Las organizaciones se desarrollan bajo una lógica de medios para el alcance de fines, los cuales pueden incorporar algunos valores; mientras que las desorganizaciones se desarrollan siguiendo una lógica de fines.

3. Las organizaciones se desarrollan sobre la base de una economía racional; mientras que las desorganizaciones se desarrollan bajo una economía del deseo y del afecto.

En función de lo expuesto, la dimensión cultural se ha configurado como un instrumento de gestión clave que promueve el mejoramiento de la productividad y la competitividad de las organizaciones en el contexto global. En esta dirección Anzola (2006) plantea que la competitividad de las empresas en los mercados globales se encuentra directamente relacionada con:

1. La cultura corporativa de la organización para adaptarse rápidamente a los cambios 
del contexto.

2. La capacidad de la organización para administrar el cambio, lo cual implica controlar el impacto y formular estrategias para encaminar las bases de la transformación hacia el alcance de los objetivos y metas de la organización.

3. La articulación de las tres subculturas que forman parte de la organización precisadas desde la postura de Schein (citado por Anzola, 2006) en la subcultura en línea que fundamenta el desarrollo del trabajo en la organización a partir de la conformación de equipos y las comunicaciones; la subcultura ingenil que promueve el mejoramiento de los productos, procesos y equipos; así como la subcultura ejecutiva que prioriza el manejo de los recursos financieros de la organización.

Así mismo, cabe acotar que la dimensión cultural presenta diferencias dentro de cada organización, pues se gesta en función de la interacción social de sus miembros. Por ende, ésta no es el resultado de la imposición de normas y reglas, sino una construcción social colectiva conformada en función de la articulación de las necesidades, objetivos y metas organizacionales con los valores, intereses y necesidades de sus miembros

Desde esta perspectiva, también resulta de interés precisar que aun cuando la cultura organizacional es estable en el tiempo para conducir al alcance de los objetivos de la organización, ésta presenta un carácter dinámico y flexible para adaptarse con facilidad a los cambios del entorno, asumiéndolos como una oportunidad para promover el desarrollo organizacional y la competitividad en los mercados globales.

A juicio de Cantillo y Daza (2011), los elementos que fomentan una cultura organizacional que promueve la competitividad se precisan en los siguientes términos:

1. El liderazgo: la consolidación y competitividad de las organizaciones en los mercados nacionales e internacionales, depende en gran medida de las estrategias adoptadas por sus líderes. En tal sentido, Castillo y Daza (2011) definen un buen líder como el actor que se compromete con la misión de la organización; comunica la visión de la empresa; tiene confianza en sí mismo, así como integridad personal. De este modo, el líder influye 
en la competitividad empresarial en la medida que sus decisiones promueven el desarrollo de la organización, a partir de la incorporación de innovaciones en productos y servicios prestados al cliente, así como en los procesos y sistemas de gestión que forman parte de la estructura de la organización.

2. Los seguidores: los empleados de la organización desempeñan una función clave para el mejoramiento de la competitividad de la organización, pues a partir de su desempeño contribuyen con el alcance de los objetivos de la organización. En tal sentido, el líder debe promover la conformación de un clima organizacional para que los empleados se identifiquen con el trabajo realizado, así como con la cultura corporativa que caracteriza la organización.

3. El capital humano: las habilidades, competencias y conocimiento de los miembros de la organización resultan un elemento clave para el mejoramiento de la competitividad de la organización. Al respecto cabe mencionar que una organización competitiva es aquella que desarrolla la capacidad para gestionar el conocimiento, lo cual depende de la existencia de una cultura organizacional que promueva el trabajo en equipo, la innovación y el desarrollo tecnológico, la participación de los miembros en la toma de decisiones, la motivación de los empleados, entre otros aspectos.

4. La productividad: el mejoramiento de la productividad se alcanza a través de un proceso continuo de mejora en el desempeño de la organización a través de la utilización óptima de los diversos factores empleados en el desarrollo del proceso productivo.

Los elementos descritos, promueven la conformación de una cultura corporativa que sustenta la competitividad de las organizaciones en los mercados globales, pues las estrategias de posicionamiento y consolidación organizacional se formulan en función de los valores, intereses y objetivos planteados para el desarrollo de la organización.

En consecuencia la integración del liderazgo, los seguidores, el capital humano y la productividad, inducen a la conformación de una cultura organizacional que conduce al alcance 
de las metas planteadas, lo cual representa una ventaja competitiva para el mejoramiento del desempeño y posicionamiento de la organización en el mercado.

De esta manera, la competitividad se entiende desde una postura que incorpora además de la dimensión económica, una de carácter cultural expresada en el consenso de los miembros de la organización sobre los principios y valores socialmente compartidos que regirán el funcionamiento organizacional para el alcance sus metas, incluyendo los aspectos relativos al posicionamiento de las organizaciones en el mercado.

Sobre la base de los argumentos expuestos, puede afirmarse que la cultura organizacional aporta las bases para la adopción de las estrategias que inducen a la conformación de una ventaja competitiva, las cuales se fundamentan en la gestión del capital humano, como elemento estratégico que promueve el desarrollo económico y social de la organización en todas sus dimensiones.

\section{Conclusiones}

La globalización es un proceso gestado a nivel mundial que ha inducido transformaciones estructurales en las diversas dimensiones del desarrollo. Estas transformaciones han influido en el funcionamiento y desempeño de las organizaciones, las cuales han adoptado nuevos modelos de gestión que han sustentado el paso de organizaciones centralizadas, jerárquicas y basadas en la división del trabajo, hacia nuevas formas organizativas de carácter centralizado, participativa, fundamentadas en el trabajo en equipo, así como en la gestión del conocimiento.

Uno de los efectos generados en el funcionamiento organizacional a partir de la globalización, se encuentra en la homogenización de los modelos de gestión, lo cual se precisa en la implementación de estructuras organizacionales homogéneas que sustentan en el caso de las Megaempresas, la producción de los mismos bienes, la prestación de los mismos servicios, la implementación de los mismos sistemas de producción, entre otros aspectos. Estas empresas, han impulsado la conformación de una cultura organizacional homogénea socialmente compartida por los miembros de la organización como base de su desarrollo a nivel global.

Sobre la base de lo expuesto, la cultura organizacional se considera actualmente como un activo estratégico de la organización que conduce al mejoramiento de su desempeño 
en los mercados globalizados. Por tanto, la dimensión cultural de la organización, entendida como una construcción social conformada a partir de los principios, creencias, valores, intereses y objetivos socialmente compartidos por los miembros de la organización, aporta una ventaja competitiva en la medida que permite el posicionamiento de las organizaciones en el mercado, el mejoramiento de su funcionamiento, así como el incremento de la productividad.

\section{Referencias bibliográficas}

Anzola, O. (2006). La cultura corporativa y su relación con la competitividad. Investigación [Revista en línea], 38-50. Disponible: http://revistas.uexternado. edu.co/index.php/sotavento/article/ viewFile/1577/1429 [Consulta: 2017, marzo, 28]

Cújar, A.; Ramos, C.; Hernández, H.; López, J. (2013). Cultura organizacional: evolución en la medición. Estudios gerenciales [Revista en linea], (29)128, 350-355. Disponible: https://www.icesi.edu.co/ revistas/index.php/estudios_gerenciales/ article/view/1720/HTML [Consulta: 2017, marzo, 28]

Chiavenato, I. (2006). Introducción a la teoría general de la administración. México: McGraw-Hill Interamericana.

Esteves, E. (2000). Globalización y empresas globales. Revista Venezolana de Análisis de Coyuntura [Revista en línea], (VI) 2, 269-280. Disponible: http://www.redalyc. org/pdf/364/36460210.pdf

Iturralde, M. (2015). Content marketing: Innovación e información a través de las redes sociales. Revista de Investigación
Sigma, 2 (01), 53 - 62.

Lanz, R. (2001). Diez tesis sobre cultura organizacional transcompleja. En R. Lanz (comp.), Organizaciones Transcomplejas (pp. 161-173). Caracas: IMPOSMOCONICIT.

Lash, S. (2005). Crítica de la información. Buenos Aires: Amorrortu Editores.

Ritzer, G. (2007). La globalización de la nada. España: Editorial popular.

Rodríguez, R. (2009). La cultura organizacional un potencial activo estratégico desde la perspectiva de la administración. Invenio [Revista en línea], (12)22, 6792. Disponible: file://C:/Users/usuario/ Downloads/Dialnet-LaCulturaOrgan izacionalUnPotencialActivoEstrateg ic-3394655.pdf [Consulta: 2017, marzo, 28]

Vargas, J. (2007). La culturocracia organizacional en México. Edición electrónica. Disponible: http://www. eumed.net/libros-gratis/2007b/301/ cultura\%20empresarial.htm 